\title{
Plasmonic Effect of Gold Nanoparticles Surrounded by Multidielectric Matrices
}

\author{
ABDUL RAUF JAMALI*, ALI DAD CHANDIO*,WASEEM KHAN*, ZUBIA ANWER*, AND \\ IMTIAZ ALI SOOMRO**
}

RECEIVED ON 18.01.2017 ACCEPTED ON 29.05.2017

\begin{abstract}
Plasmonic materials are the artificially manufactured materials (metamaterials) typically composed of nanostructure noble metals which give the unique optical properties superior to naturally occurring materials. Such plasmonic material has ability to give surface plasmon when electromagnetic light wave interact with free electrons in conduction band of the noble metals. Entirely absorption of light wave is the one of the major property of such plasmonicmetamaterials. Recently, these metamaterials has drawn the attention of scientists because of its wide range of applications in harvesting energy and sensors. These applications are strongly dependent on adjusting the absorption band-width, peak position and absorption intensity. In this work we show that using ultra-thin multicomponent (i.e. Au NPs incorporated in mixture of $\mathrm{SiO}_{2}$ and $\mathrm{TiO}_{2}$ matrix) nanocomposite deposited on optically thick gold film through subsequent magnetron sputtering. Experimental results show that one can broaden the bandwidth and peak position to span Ultraviolet up to near infrared wavelength range.
\end{abstract}

Key Words: Super-Absorber, Multicomponent Nanocomposite, Plasmonics, Metamaterials.

\section{INTRODUCTION}

$\mathrm{N}$

early perfect absorption of electromagnetic light wave from range of visible to near-

infrared frequencies is one of the recent most important properties of material which has broadened in its applications. From last year till to date, the developments to achieve perfect absorbers increased enormously because of its enormous interest in the development of material for solar energy for harvesting and sensors applications. With concern to the wide application range of perfect absorber, improved fabrication methods (such as perforated metallic films, grating structured systems, and metamaterials) have also been taken into account till to date [1]. Out of different materials and composites which are limited by lack of stability, cost and also limited narrow absorbance spectral range, multi-component plasmonic nanocomposite where noble metal NPs (Nanoparticles) surrounded by dielectric matrices in the form of nanocomposites have gained significant research interest on experimental as well as theoretical basis due their varietyof properties appropriate for various applications ranging from solar cells to biosensing devices. Furthermore, their absorbance spectra at broad range of frequencies could also be possible [1].

\footnotetext{
* Department Metallurgical Engineering, NED University of Engineering \& Technology, Karachi.

** Department of Metallurgy \& Materials Engineering, Mehran University of Engineering \& Technology, Jamshoro.
} 
The aim of this research work is to experimentally fabricate and characterize the optical properties of the three components super-absorber structure. Another aim is to investigate perfect absorption behavior in wide frequency range. For this purposes, we use gold NPs (as a filler material) surrounded by $\mathrm{TiO}_{2}$ and $\mathrm{SiO}_{2}$ matrices (as dielectric environment). The absorption peak of depends on different materials parameters (such as dielectric environment, filling factor ff and nanocomposite film thickness d). Increasing and decreasing refractive index (dielectric ratio) allow us to shift absorption peak from short to long wavelength ranges, respectively. Considering the index of refraction (material property), multicomponent (having different refractive indices) is used. The plasmonic properties of the nanocomposite films mainly determine by the type of noble metal (gold) NPs, their morphology and the dielectric constant of the embedding matrix. In dielectric constant, the refractive index of the matrices in nanocomposite plays a very important role in SPR (Surface Plasmon Resonance). Insulating matrices, such as $\mathrm{SiO}_{2}, \mathrm{TiO}_{2}$ and polymers can be used to enhance the properties of plasmonic materials for different applications. The main reason behind the use of insulating matrices is to maintain necessary separation metallic NPs and also to avoid possible agglomeration of metallic NPs [2] and hence obtain an effective plasmonic resonance of metallic NPs and thereby SPP.

\section{EXPERIMENTAL WORK}

The physical vapor deposition of the gold film (on glass substrate) and entire nanocomposite (on $100 \mathrm{~nm}$ gold) were performed by a magnetron sputtering machine. Fig. 1 shows the arrangement of functional layers on glass substrate.

Three magnetron targets (two RF sources and one DC source) can be run independently with rotatable sample holder in order to achieve uniform distribution of the components and homogeneous film thickness. The schematic design of the magnetron sputtering process is shown in Fig. 2.

Based on the experimental results, it seems that the absorption efficiency of nanocomposite is mainly depends on three factors i.e. dielectric ratio, volume filling factor $\mathbf{f f}$ and the nanocomposite film thickness $\mathbf{d}$. The volume filling factor of the nanocomposite is estimated based on the rate of deposition. UV-Vis-NIR spectrometer (LAMBDA900-PerkinElmer) was used to collect the reflection (R) and transmission $(\mathrm{T})$ of the samples and absorption (A) having no scattering, can be calculated

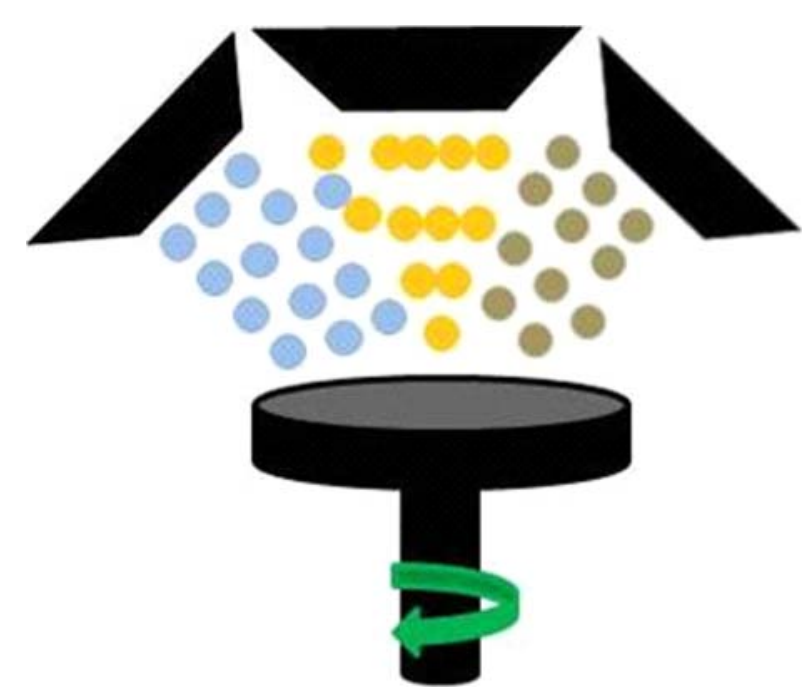

FIG. 1. SKETCH OF MAGNETRON SPUTTERING (WITH THREE MAGNETRONS TRAGETS). SAMPLE IS PLACED UNDER THESE TARGETS AND ROTATING WITH CONSTANT SPEED

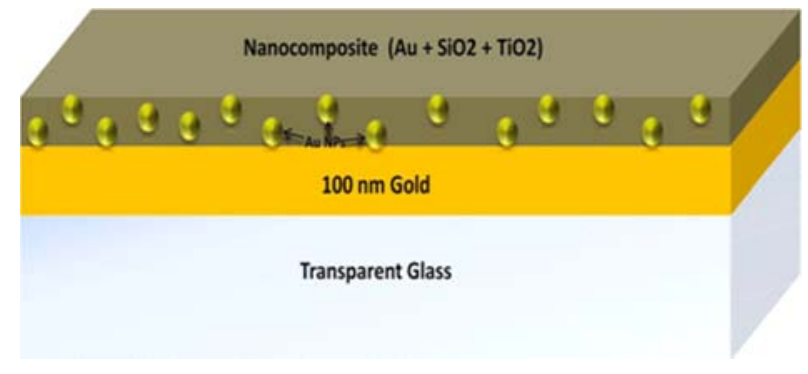

FIG. 2. SCHEMATIC OF THE GLASS SUBSTRATE COMPOSED OF THICK GOLD FILM AND NANOCOMPOSITE ON THE TOP

Mehran University Research Journal of Engineering \& Technology, Volume 36, No. 3, July, 2017 [p-ISSN: 0254-7821, e-ISSN: 2413-7219] 
directly from transmittance (T) and reactance (R) using Kirchhoff's rule (A= 1-T-R). Film thickness and angle of refraction analysis of the samples were carried out with an automated angle M-2000® Ellipsometer (JAWoollam Co.).However, profilometry (Dektak XT-E-BRUKER) is also applied for gold film thickness measurements.

\section{RESULTS AND DISCUSSION}

Following previous work $[1,3]$, it is known that the contribution of index of refraction may alter the absorption peak from short to long wavelength. Fig. 3 shows the absorption spectra of the nanocomposite deposited on optically thick gold film (100 nm Au film) with altered dielectric ratios $\left(\mathrm{SiO}_{2}: \mathrm{TiO}_{2}\right)$. It is seen in Fig. 3 that by increasing the percentage of $\mathrm{TiO}_{2}$ (having higher refractive index $=2.5$ ) in nanocomposite, the absorption peak is shifting to longer wavelengths and its intensity going to drop. While by increasing the percentage of $\mathrm{SiO}_{2}$ (having lower refractive index $=1.5$ ), the absorption peak is shifting to shorter wavelengths. For higher percentage of $\mathrm{TiO}_{2}$ (having higher refractive index) the retardation effect on

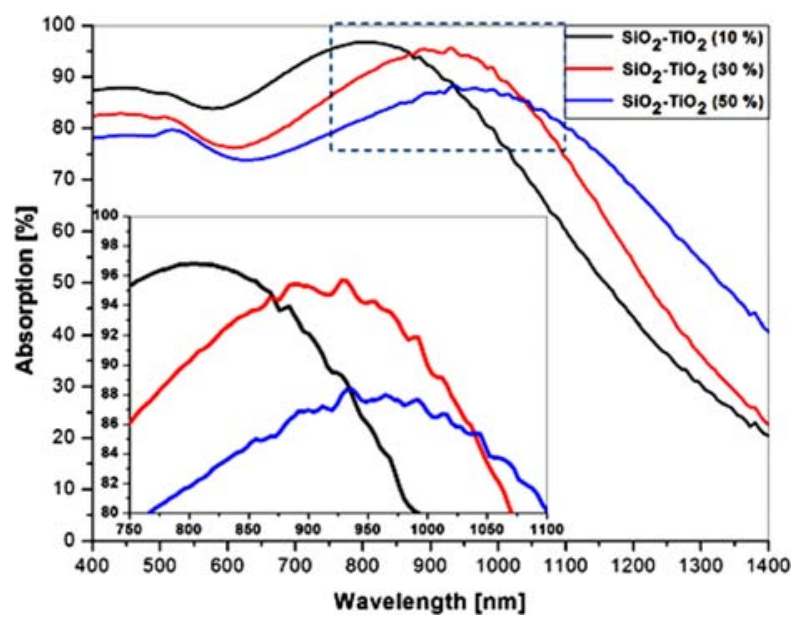

FIG. 3. UV-VIS ABSORPTION SPECTRA FOR NANOCOMPOSITE DEPOSITED ONTHICK METALLIC FILM (100 NM AU FILM) WITH VARIOUS DIELECTRIC RATIOS 10\% $\mathrm{TIO}_{2}: 90 \% \mathrm{SIO}_{2}$ (BLACK CURVE), 30\% $\mathrm{TIO}_{2}: 70 \% \mathrm{SIO}_{2}$ (RED CURVE) AND 50\% $\mathrm{TIO}_{2}: 50 \% \mathrm{SIO}_{2}$ (BLUE CURVE) WHILE GOLD FILLING FACTOR (IE FF $=40 \%$ ) ANDNANOCOMPOSITE FILM THICKNESS (IE D = $50 \mathrm{NM}$ ) KEPT CONSTANT. INSET IS THEMAGNIFIED PLOT OF DASHED AREA the resonance of the metallic particles is shifting the absorption band to a longer wavelengths [3]. While the absorption band to shorter wavelengths shift for higher percentage of $\mathrm{SiO}_{2}$ is attributed to the higher resonance frequency for metallic particles in low dielectric medium is more dominant [1].

In order to maintain higher absorption intensity from VIS (Vaccine Statements) to NIR (Near-Infrared) frequency range, the dielectric matrices of $\mathrm{SiO}_{2}$ as well as $\mathrm{TiO}_{2}$ is adjusted at optimum ratio. From number of experiments, it is realized that $50 \% \mathrm{TiO}_{2}$ and $50 \% \mathrm{SiO}_{2}$ is the optimum condition for observing super-absorption as shown in Fig. 3. If the percentage of $\mathrm{TiO}_{2}$ will go beyond the optimum condition i.e. $50 \%$ of $\mathrm{TiO}_{2}$, one could not get perfect absorption (around 100\%). From Fig. 3, one can see that at higher frequency the absorption for optimum condition (50\% $\mathrm{TiO}_{2}$ and $50 \% \mathrm{SiO}_{2}$ ) innanocomposite is higher as compare to other dielectric ratios. This could be attribute to the greater percentage of dielectric constant (having high refractive index) in nanocomposite disturbs the condition of impedance matching to the vacuum and hence absorption decreases [1]. This is because of the contribution of high refractive index materiel is more innanocomposite. Here the absorption dip is also very small and can be neglected. Hence the characteristic perfect absorption at effective dielectric ratios can also be seen from 400-1400 nm frequency in Fig. 3.

The microstructural morphologies of $\mathrm{SiO}_{2}-\mathrm{TiO}_{2}$ composites were investigated byTEM (Transmission Electron Microscopy) and are shown in Fig. 4. In Fig. 4 (Left), the bright field TEM image for nanocomposite where uniform distribution of $\mathrm{SiO}_{2}$ and $\mathrm{TiO}_{2}$ matrices in nanocompostie were observed, black and white spots correspond to the $\mathrm{SiO}_{2}$ matrix and the $\mathrm{TiO}_{2}$ matrix, respectively [4]. The SAED (Selected Area Electron Diffraction) patternsof nanocomposite film are shown right side of the bright-field TEM image. In Fig. 4 (Right), SAED pattern demonstrate that the $\mathrm{TiO}_{2}-\mathrm{SiO}_{2}$ matrices in the nanocomposite film are in an amorphous state. 

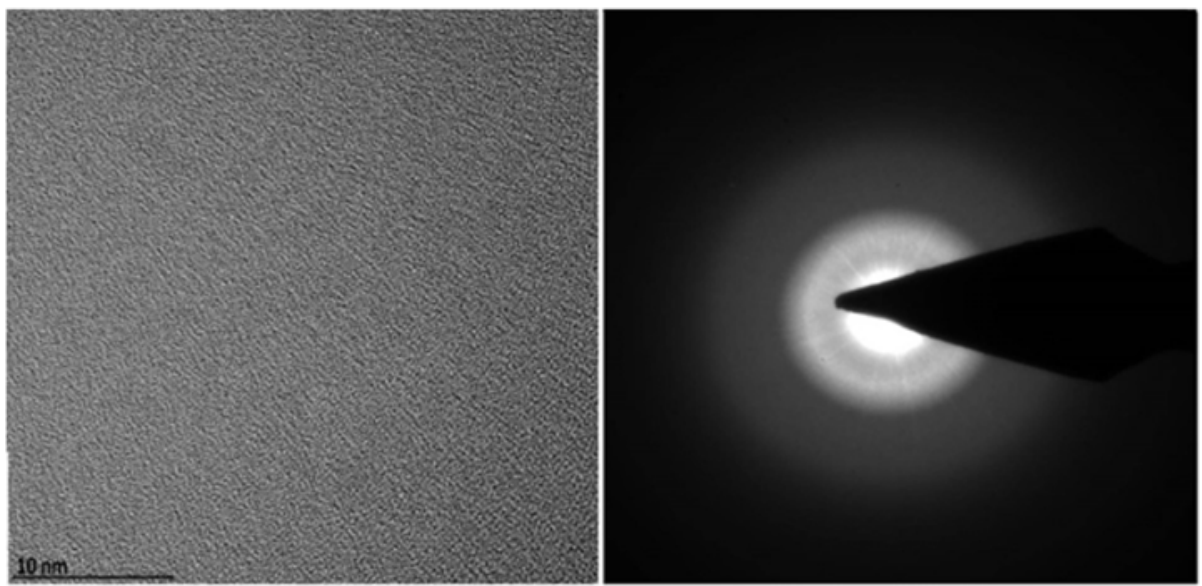

FIG. 4. BRIGHT-FIELD TEM IMAGE (LEFT) OF TIO ${ }_{2}$ SIO $_{2}$ NANOCOMPOSITE. SIO MATRIX CAN BE SEEN BY DARK WHILE TIO MATRIX CAN BE SEEN BY BRIGHT AREA. THE SELECTED AREA ELECTRON DIFFRACTION (SAED) PATTERNS FOR THIN FILM NANOCOMPOSITE ARE SHOW ON RIGHT SIDE OF TEM IMAGE

\section{CONCLUSION}

We have fabricated a two functional layers perfect absorber by using a thick metallic film (100 nm gold film) and three components nanocomposite $\left(\mathrm{SiO}_{2}-\right.$ $\mathrm{Au}-\mathrm{TiO}_{2}$ ). The fabrication technique is done by PVD magnetron sputtering. Adjusting dielectric ratio using different dielectric materials, we are able to tune our perfect absorber for wide range of wavelength spectrum.

\section{ACKNOWLEDGEMENTS}

The authors would like to pay many thanks to Nanochemistry and Nanoengineering Group, University of Kiel, Germany, for providing access to all lab facilities. Authors also wish to thank the Organizing Committee of $2^{\text {nd }}$ National Conference on Metallurgy \& Materials, for providing the platform for oral presentation and publishing the paper in Mehran University Research Journal of Engineering \& Technology.

\section{REFERENCES}

[1] Hedayati, M.K., Faupel, F., Elbahri, M.,Javaherirahim, M., Mozooni,B., Abdelaziz, R., Tavassolizadeh,A., Chakravadhanula, V.S., Zaporojtchenko, V., and Strunkus, T., "Design of a Perfect Black Absorber at Visible Frequencies using PlasmonicMetamaterials”, Advanced Materials, Volume 23, pp. 5410-5414, 2011.
[2] Chakravadhanula, V., Mishra, Y., Kotnur, V., Avasthi, D., Strunskus, T., Zaporotchenko, V., Fink, D., Kienle, L., and Faupel, F., "Microstructuraland Plasmonic Modi_Cations in $\mathrm{Ag}-\mathrm{TiO}_{2}$ and $\mathrm{Au}-\mathrm{TiO}_{2}$ Nanocomposites through Ion Beam Irradiation”,Beilstein Journal of Nanotechnology, Volume 5, pp. 1419-1431, 2014.

[3] Hedayati, M.K., Faupel, F., and Elbahri, M., "Review of Plasmonic Nanocomposite Metamaterial Absorber”, Materials, Volume 7, pp. 1221-1248, 2014.

[4] Wu, Y., Yu, L., and Zhi, J., "Low Cost and Large-Area Fabricationof Self-Cleaning Coating on Polymeric Surface Based on Electrolessplating-Like Solution Deposition Approach”, The Royal Society of Chemistry, 2015.

[5] Hedayati, M.K., Javaheri, M., Zillohu, A.U., ElKhozondar, H.J., Bawa’aneh, M.S.L.A., Faupel, F., and Elbahri, M., "Photo-Driven Super Absorber as an Active Metamaterial”, Advanced Optical Materials, Volume 2, No. 8, pp. 705-710,2014.

[6] Fahr, S., Etrich, C., Faupel, F., Rockstuhl, C., and Elbahri, M., "The Hybrid Concept for Realization of an UltraThin Plasmonic Metamaterial Antireflection Coating and Plasmonic Rainbow”, Nanoscale, Volume 6, No. 11, pp. 6037-6045,2014. 